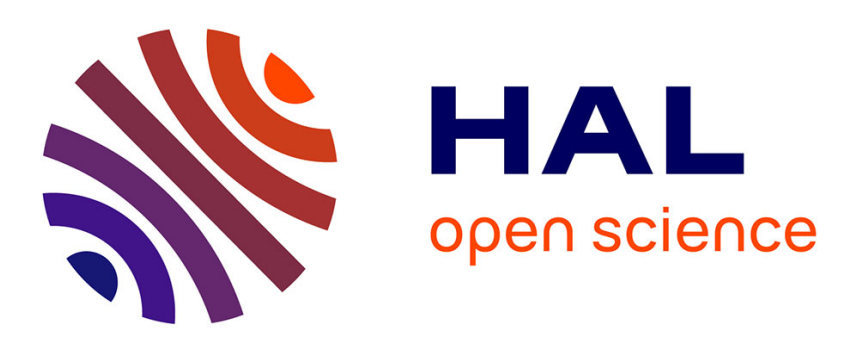

\title{
Invariant subgrid modelling in large-eddy simulation of heat convection turbulence
}

Dina Razafindralandy, Aziz Hamdouni

\section{To cite this version:}

Dina Razafindralandy, Aziz Hamdouni. Invariant subgrid modelling in large-eddy simulation of heat convection turbulence. Theoretical and Computational Fluid Dynamics, 2007, 21 (4), pp.231-244. 10.1007/s00162-007-0046-1 . hal-00312284

\section{HAL Id: hal-00312284 \\ https://hal.science/hal-00312284}

Submitted on 1 Apr 2019

HAL is a multi-disciplinary open access archive for the deposit and dissemination of scientific research documents, whether they are published or not. The documents may come from teaching and research institutions in France or abroad, or from public or private research centers.
L'archive ouverte pluridisciplinaire HAL, est destinée au dépôt et à la diffusion de documents scientifiques de niveau recherche, publiés ou non, émanant des établissements d'enseignement et de recherche français ou étrangers, des laboratoires publics ou privés. 


\title{
Invariant subgrid modelling in large-eddy simulation of heat convection turbulence
}

Received: date / Accepted: date

\begin{abstract}
Symmetries have an important role in turbulence. To some extent, they contain the physics of the equations (conservation laws,...), and it is essential that turbulence models respect them. However, as observed by Oberlack ([16]) and next by Razafindralandy and Hamdouni ([24]) in the case of an isothermal fluid, only few subgrid stress tensor models preserve the symmetries of the Navier-Stokes equations. In this communication, we present the symmetries of the equations of a non-isothermal fluid flow and analyze some common subgrid stress tensor and flux models under the point of view of these symmetries.
\end{abstract}

Keywords Turbulence · Large-eddy simulation · Heat convection · Symmetry group

PACS 47.27.E- - 47.27.ep

\section{Introduction}

For some decades, an important effort has been dedicated to the derivation of turbulence models for the simulation of fluid flows. This was done using various concepts and hypothesis. However, these derivations did not take into consideration the invariance of the base equations under some transformations, called symmetries, which traduces fundamental properties of the flow.

A symmetry of an equation is a transformation which takes a solution of the equation into another solution. In other words, it is a transformation which preserves the set of the solutions. Symmetries play an important role in mechanics. Indeed, for example, Nother's theorem $([15,19])$ show how each symmetry of a Lagrangian corresponds to a conservation law. So, if any time translation is a symmetry of the Lagrangian then the energy is conserved along the solution path. In the same way, if the spatial translations are symmetries of the Lagrangian then the linear momentum is a conserved quantity, etc. In turbulence, even if the Navier-Stokes equations can not be directly written in a Lagrangian form, Nœether's theorem can be applied to them by extending the notion of Lagrangian ([23]).

The importance of symmetries in turbulence is not limited to the derivation of conservation laws. Indeed, scaling transformations, being part of the symmetry set of the Navier-Stokes equations, led Oberlack ([17]) to calculate scaling laws, such as algebraic and logarithmic laws. These transformations also permitted Ünal ([28]) to show that the Navier-Stokes equations may admit solutions having the $5 / 3$ Kolmogorov spectra. The theory of symmetry groups permits even to calculate exact solutions ([19, 6]). Next, some self-similar solutions, i.e. solutions which are not altered by a symmetry transformation, give an information on the behaviour of the solutions at a very large time (see [2]). Finally, we mention

D. Razafindralandy, A. Hamdouni

LEPTAB, Avenue Michel Crépeau, 17042 La Rochelle Cedex 1

Tel.: +33-5 46458332

Fax: +33-5 46458241

E-mail: drazafin@univ-lr.fr, ahamdoun@univ-lr.fr 
that the use of discretization schemes which are compatible with the symmetries of an equation reduces the numerical errors $([20,12])$.

The introduction of a turbulence model in the equations of a flow may destroy the symmetry invariance of these equations. In this case, physical properties (conservation laws, scaling laws, spectral properties, large-time behaviour, ...) may be lost.

It is accepted for a long time that Galilean invariance, which is a symmetry of the Navier-Stokes equations, should be preserved by any turbulence model. Later, Speziale ([27]) was the first who recognized that the two-dimensional material indifference, which is an additional symmetry of the equations, is a key property that any subgrid-scale model should have. He has been followed by many authors such that Fureby and Tabor ([5]) and Wang ([30,31]). The first author who considered all the symmetries of the isothermal Navier-Stokes equations in the analysis of turbulence models for an isothermal fluid is Oberlack ([16]), followed by Razafindralandy and Hamdouni ([24,26]). Oberlack analyzed some subgrid stress tensor models and showed that only few of them are compatible with the symmetries of the Navier-Stokes equations. Razafindralandy and Hamdouni drew the same conclusion when extending the analysis to other common subgrid stress models. This incited them to derive new subgrid stress tensor models which are consistent with the symmetries of the Navier-Stokes equations $([25,24])$.

In the case of a non-isothermal flow, no analysis has yet been done. Though, such an analysis is indispensable, in order to know if we can hope that the current existing models are able to reproduce correctly the flow or if, on the contrary, it is necessary to derive new models which are consistent with the symmetries of the equations. The aim of this article is then to present the symmetries of the Navier-Stokes equations coupled with the heat convection equations, and to extend the analysis done in [24] to the case of a non-isothermal fluid flow. In section 2, the symmetries of the equations are presented, and in section 3, some common subgrid stress tensor and heat flux models are recalled and analyzed by the symmetry approach.

\section{The symmetry group of the non-isothermal Navier-Stokes equations}

Consider an incompressible Newtonian fluid of density $\rho$, kinematic viscosity $\nu$, thermal expansion coefficient $\beta$ and thermal diffusivity $\kappa$. Let $g$ be the gravity acceleration and $\mathbf{e}_{3}$ the ascending vertical unitary vector. If we note $t$ the time variable, $\mathbf{x}=\left(x_{i}\right)_{i=1,2,3}$ the space variable, $\mathbf{u}=\left(u_{i}\right)_{i}$ the velocity field, $p$ the pressure and $\theta$ the temperature then the motion of this fluid is governed by the nonisothermal Navier-Stokes equations:

$$
\left\{\begin{array}{l}
\frac{\partial \mathbf{u}}{\partial t}+\operatorname{div}(\mathbf{u} \otimes \mathbf{u})+\frac{1}{\rho} \nabla p-\operatorname{div} \boldsymbol{T}_{r}-\beta g \theta \mathbf{e}_{3}=0 \\
\frac{\partial \theta}{\partial t}+\operatorname{div}(\theta \mathbf{u})-\operatorname{div} \mathbf{h}_{r}=0 \\
\operatorname{div} \mathbf{u}=0
\end{array}\right.
$$

In these expressions, $\boldsymbol{T}_{r}=2 \nu \boldsymbol{S}$ is the viscous stress tensor, $\boldsymbol{S}=\left[\nabla \mathbf{u}+{ }^{T} \nabla \mathbf{u}\right] / 2$ the strain rate tensor and $\mathbf{h}_{r}=\kappa \nabla \theta$ the heat flux. For simplicity, let us abbreviate (1) by

$E(\boldsymbol{\xi}, \mathbf{w})=0$

where $\boldsymbol{\xi}=\left(\boldsymbol{\xi}_{i}\right)_{i}=(t, \mathbf{x})$ is the independent variable and $\mathbf{w}=\left(w_{i}\right)_{i}=(\mathbf{u}, p, \theta)$ the dependent variable.

A one-parameter point transformation is a transformation

$T_{a}: \mathbf{q}=(\boldsymbol{\xi}, \mathbf{w}) \longmapsto \widehat{\mathbf{q}}=(\widehat{\boldsymbol{\xi}}, \widehat{\mathbf{w}})$

where $\widehat{\mathbf{q}}=\widehat{\mathbf{q}}(\mathbf{q}, a)$ depends continuously on the real parameter $a$. This transformation is a symmetry of (2) if, to each solution of (2), it corresponds another solution, i.e.

$E(\boldsymbol{\xi}, \mathbf{w})=0 \quad \Longrightarrow \quad E(\widehat{\boldsymbol{\xi}}, \widehat{\mathbf{w}})=0$. 
The set of the one-parameter symmetries of (2) constitutes a (local) group

$G=\left\{T_{a}, a \in I \subset \mathbb{R}\right\}$

called a symmetry group of (2). $I$ is an interval of $\mathbb{R}$. Assume that the identity element of this group corresponds to $a=0$ (i.e. the group is additive). This group is then characterized by its variation near $a=0$ which is represented by the vector field or infinitesimal generator

$\mathbf{X}=\sum_{m} X^{m} \frac{\partial}{\partial q_{m}}, \quad$ where $X^{m}=\left.\frac{\partial \widehat{q}_{m}}{\partial a}\right|_{a=0}$

The $q_{m}$ 's and $\widehat{q}_{m}$ 's are respectively the components of $\mathbf{q}$ and $\widehat{\mathbf{q}}$. More precisely, $\mathbf{X}$ is defined by:

$f \in C^{\infty} \mapsto \mathbf{X} . f=\sum_{m} X^{m} \frac{\partial f}{\partial q_{m}}$.

The knowledge of $\mathbf{X}$ involves the determination of the symmetry group. Indeed, knowing the $X^{m}$ 's, the expression of $\widehat{\mathbf{q}}$ can be obtained from the definition of $X^{m}$ in (6) together with the (initial) condition

$\widehat{\mathbf{q}}(\mathbf{q}, 0)=\mathbf{q}$.

Lie's theory (see $[19,9]$ ) permits to replace the symmetry condition (4) by the following one which is easier to handle:

$E(\mathbf{q})=0 \quad \Longrightarrow \quad \mathbf{X} \cdot E=0$.

In the right-side equation in (9), $\mathbf{X}$ is prolonged to take into account the derivative terms in $E$ (see, for example, (67)). The resolution of (9) leads to the infinitesimal generators of (2) and then to all the one-parameter symmetries.

The calculation of the infinitesimal generators of (2) is too long to be done manually but since all the operations are formal, it can be automatized thanks to symbolic computation softwares $([7,10])$. However, as an example, the infinitesimal generators and the symmetries of the convection-diffusion equation are calculated manually in appendix $\mathrm{A}$.

Using the Maple package DESOLV of Vu and Carminatti ([29]), we could compute the infinitesimal generators of $(1)$, corresponding to transformations of the form

$(t, \mathbf{x}, \mathbf{u}, p, \theta) \longmapsto(\widehat{t}, \widehat{\mathbf{x}}, \widehat{\mathbf{u}}, \widehat{p}, \widehat{\theta})$.

These generators are:

$$
\begin{aligned}
& \mathbf{X}_{1}=\frac{\partial}{\partial t} \\
& \mathbf{X}_{2}=\zeta(t) \frac{\partial}{\partial p} \\
& \mathbf{X}_{3}=\beta g x_{3} \frac{\partial}{\partial p}+\frac{1}{\rho} \frac{\partial}{\partial \theta} \\
& \mathbf{X}_{4}=x_{2} \frac{\partial}{\partial x_{1}}-x_{1} \frac{\partial}{\partial x_{2}}+u_{2} \frac{\partial}{\partial u_{1}}-u_{1} \frac{\partial}{\partial u_{2}}, \\
& \mathbf{X}_{4+i}=\alpha_{i}(t) \frac{\partial}{\partial x_{i}}+\dot{\alpha}_{i}(t) \frac{\partial}{\partial u_{i}}-\rho x_{i} \ddot{\alpha}_{i}(t) \frac{\partial}{\partial p} \\
& \mathbf{X}_{8}=2 t \frac{\partial}{\partial t}+\sum_{j=1}^{3} x_{j} \frac{\partial}{\partial x_{j}}-\sum_{j=1}^{3} u_{j} \frac{\partial}{\partial u_{j}}-2 p \frac{\partial}{\partial p}-3 \theta \frac{\partial}{\partial \theta} \quad i=1,2,3,
\end{aligned}
$$

where $\zeta$ and the $\alpha_{i}$ 's are arbitrary functions of time. The dot symbol $\left({ }^{*}\right)$ stands for derivation. 
We can also consider symmetries (which are sometimes called equivalence transformations) of the form

$(t, \mathbf{x}, \mathbf{u}, p, \theta, \nu, \kappa) \longmapsto(\widehat{t}, \widehat{\mathbf{x}}, \widehat{\mathbf{u}}, \widehat{p}, \widehat{\theta}, \widehat{\nu}, \widehat{\kappa})$.

Such symmetries take a solution of (1) into a solution of other non-isothermal Navier-Stokes equations with a different value of $\nu$ and $\kappa$. Again using the package DESOLV, we obtain an additional infinitesimal generator

$\mathbf{X}_{9}=\sum_{j=1}^{3} x_{j} \frac{\partial}{\partial x_{j}}+\sum_{j=1}^{3} u_{j} \frac{\partial}{\partial u_{j}}+2 p \frac{\partial}{\partial p}+\theta \frac{\partial}{\partial \theta}+2 \nu \frac{\partial}{\partial \nu}+2 \kappa \frac{\partial}{\partial \kappa}$

With these generators, we deduce the symmetry groups of (1) which are:

- the group of time translations, corresponding to $\mathbf{X}_{1}$ :

$$
(t, \mathbf{x}, \mathbf{u}, p, \theta) \longmapsto(t+a, \mathbf{x}, \mathbf{u}, p, \theta),
$$

- the group of pressure translations, corresponding to $\mathbf{X}_{2}$ :

$$
(t, \mathbf{x}, \mathbf{u}, p, \theta) \longmapsto(t, \mathbf{x}, \mathbf{u}, p+\zeta(t), \theta),
$$

- the group of pressure-temperature translations, corresponding to $\mathbf{X}_{3}$ :

$$
(t, \mathbf{x}, \mathbf{u}, p, \theta) \longmapsto\left(t, \mathbf{x}, \mathbf{u}, p+a \beta g x_{3}, \theta+a \frac{1}{\rho}\right)
$$

- the group of horizontal rotations, corresponding to $\mathbf{X}_{4}$ :

$(t, \mathbf{x}, \mathbf{u}, p, \theta) \longmapsto(t, \boldsymbol{R} \mathbf{x}, \boldsymbol{R u}, p, \theta)$

where $\boldsymbol{R}$ is a $2 \mathrm{D}$ (constant) rotation matrix, with $\boldsymbol{R}^{T} \boldsymbol{R}=\boldsymbol{I}_{d}$ and $\operatorname{det} \boldsymbol{R}=1, \boldsymbol{I}_{d}$ being the identity matrix,

- the (three-parameter) group of generalized Galilean transformations, spanned by the $\mathbf{X}_{4+i}$ 's, $i=$ $1,2,3$ :

$$
(t, \mathbf{x}, \mathbf{u}, p, \theta) \longmapsto(t, \mathbf{x}+\boldsymbol{\alpha}(t), \mathbf{u}+\dot{\boldsymbol{\alpha}}(t), p+\rho \mathbf{x} \cdot \ddot{\boldsymbol{\alpha}}(t), \theta),
$$

- the group of the first scaling transformations generated by $\mathbf{X}_{8}$ :

$$
(t, \mathbf{x}, \mathbf{u}, p, \theta) \longmapsto\left(\mathrm{e}^{2 a} t, \mathrm{e}^{a} \mathbf{x}, \mathrm{e}^{-a} \mathbf{u}, \mathrm{e}^{-2 a} p, \mathrm{e}^{-3 a} \theta\right)
$$

which shows how $\mathbf{u}, p$ and $\theta$ change when the spatio-temporal scale is multiplied by $\left(\mathrm{e}^{a}, \mathrm{e}^{2 a}\right)$,

- and the group of the second scaling transformations corresponding to $\mathbf{X}_{9}$ :

$$
(t, \mathbf{x}, \mathbf{u}, p, \theta, \nu, \kappa) \longmapsto\left(t, \mathrm{e}^{a} \mathbf{x}, \mathrm{e}^{a} \mathbf{u}, \mathrm{e}^{2 a} p, \mathrm{e}^{a} \theta, \mathrm{e}^{2 a} \nu, \mathrm{e}^{2 a} \kappa\right)
$$

which shows the consequence of the modification of the spatial scale.

The space translations correspond to transformations (23) where $\boldsymbol{\alpha}=\left(\alpha_{i}\right)_{i}$ is constant and the classical Galilean transformation to the case where $\boldsymbol{\alpha}$ is linear.

Other known symmetries of the non-isothermal Navier-Stokes equations (1), which could not be computed like the previous ones, are 
- the reflections:

$(t, \mathbf{x}, \mathbf{u}, p, \theta) \mapsto\left(t, \boldsymbol{\Lambda} \mathbf{x}, \boldsymbol{\Lambda} \mathbf{u}, p, \iota_{3} \theta\right)$

where $\boldsymbol{\Lambda}$ is a reflection matrix:

$$
\boldsymbol{\Lambda}=\left(\begin{array}{ccc}
\iota_{1} & 0 & 0 \\
0 & \iota_{2} & 0 \\
0 & 0 & \iota_{3}
\end{array}\right) \quad \text { with } \quad \iota_{i}= \pm 1, \quad i=1,2,3
$$

- and the material indifference in the limit of a 2D horizontal flow in a simply connected domain ([3]) which is a time-dependent rotation:

$(t, \mathbf{x}, \mathbf{u}, p) \mapsto(t, \widehat{\mathbf{x}}, \widehat{\mathbf{u}}, \widehat{p})$,

with

$\widehat{\mathbf{x}}=\boldsymbol{R}(t) \mathbf{x}, \quad \widehat{\mathbf{u}}=\boldsymbol{R}(t) \mathbf{u}+\dot{\boldsymbol{R}}(t) \mathbf{x}, \quad \widehat{p}=p-3 \omega \varphi+\frac{1}{2} \omega^{2}\|\mathbf{x}\|^{2}$

where $\boldsymbol{R}(t)$ is an horizontal 2D rotation matrix with angle $\omega t, \omega$ an arbitrary real constant, $\varphi$ the usual $2 \mathrm{D}$ stream function defined by:

$\mathbf{u}=\operatorname{curl}\left(\varphi \mathbf{e}_{3}\right)$

and $\|\bullet\|$ indicates the Euclidian norm.

The combination of all these symmetries constitutes a group, called the symmetry group of the nonisothermal Navier-Stokes equations. Compared to the case of the isothermal Navier-Stokes equations ([22]), we have new symmetries which are the pressure-temperature translations (21). Note that the vertical reflection (corresponding to $\iota_{3}=-1$ in (26)) stops to be a symmetry of the equations if we introduce the condition that $\theta \geq 0$.

For the model analysis, the symmetries will be gathered in four categories:

- the time, the pressure and the generalized Galilean translations,

- the pressure-temperature translations,

- the reflections, the horizontal (constant or time-dependent) rotations,

- the scaling transformations.

\section{Model analysis}

In this section, some subgrid turbulence models are briefly recalled and analyzed according to the symmetry approach.

After filtering, the large-eddy simulation method gives

$$
\left\{\begin{array}{l}
\frac{\partial \overline{\mathbf{u}}}{\partial t}+\operatorname{div}(\overline{\mathbf{u}} \otimes \overline{\mathbf{u}})+\frac{1}{\rho} \nabla \bar{p}-\operatorname{div}\left(\overline{\boldsymbol{T}}_{r}-\boldsymbol{T}\right)-\beta g \bar{\theta} \mathbf{e}_{3}=0 \\
\frac{\partial \bar{\theta}}{\partial t}+\operatorname{div}(\bar{\theta} \overline{\mathbf{u}})-\operatorname{div}\left(\overline{\mathbf{h}}_{r}-\mathbf{h}\right)=0 \\
\operatorname{div} \overline{\mathbf{u}}=0 .
\end{array}\right.
$$

The symbol bar $\left(^{-}\right)$stands for filtering, with a width $\bar{\delta} . \boldsymbol{T}=\overline{\mathbf{u} \otimes \mathbf{u}}-\overline{\mathbf{u}} \otimes \overline{\mathbf{u}}$ is the subgrid stress tensor and $\mathbf{h}=\overline{\theta \mathbf{u}}-\bar{\theta} \overline{\mathbf{u}}$ the subgrid flux. These two terms must be modeled in order to close the equations. 
3.1 Subgrid models

Some subgrid models are summarized in this subsection.

- The most widely used model is the Smagorinsky model which was derived by adopting the concept of turbulent viscosity for $\boldsymbol{T}$ and an analogy for $\mathbf{h}$ :

$$
\boldsymbol{T}^{d}=-2\left(C_{s} \bar{\delta}\right)^{2}|\overline{\boldsymbol{S}}| \overline{\boldsymbol{S}}, \quad \mathbf{h}=-\frac{\left(C_{s} \bar{\delta}\right)^{2}}{P r_{s g}}|\overline{\boldsymbol{S}}| \nabla \bar{\theta},
$$

where

$$
|\overline{\boldsymbol{S}}|=\sqrt{2 \sum_{i j} \overline{\boldsymbol{S}}_{i j}^{2}}
$$

$C_{s}$, the Smagorinsky constant, and $P r_{s g}$, the subgrid Prandtl number, are constant. The superscript $d$ stands for the deviatoric operator, i.e.:

$\boldsymbol{T}^{d}=\boldsymbol{T}-\left(\frac{1}{3} \operatorname{tr} \boldsymbol{T}\right) \boldsymbol{I}_{d}$

$\boldsymbol{I}_{d}$ being the identity matrix.

- By using Germano-Lilly procedure ([13]), the model constants can be calculated in a dynamic way. This leads to the dynamic model:

$$
\begin{aligned}
& \boldsymbol{T}^{d}=-2 C_{\boldsymbol{T}} \bar{\delta}^{2}|\overline{\boldsymbol{S}}| \overline{\boldsymbol{S}}, \quad \mathbf{h}=-C_{\mathbf{h}} \bar{\delta}^{2}|\overline{\boldsymbol{S}}| \nabla \bar{\theta} \\
& \text { where } C_{\boldsymbol{T}}=\operatorname{tr}(\boldsymbol{L} \boldsymbol{M}) / \operatorname{tr}\left(\boldsymbol{M}^{2}\right), C_{\mathbf{h}}=\operatorname{tr}\left(\mathbf{L}^{\prime}{ }^{T} \mathbf{M}^{\prime}\right) / \operatorname{tr}\left(\mathbf{M}^{\prime}{ }^{T} \mathbf{M}^{\prime}\right) \text { and } \\
& \boldsymbol{L}=\widetilde{\overline{\mathbf{u}} \otimes \overline{\mathbf{u}}}-\widetilde{\overline{\mathbf{u}}} \otimes \widetilde{\overline{\mathbf{u}}}, \quad \boldsymbol{M}=\widetilde{\bar{\delta}}^{2}|\widetilde{\bar{S}}| \widetilde{\bar{S}}-\bar{\delta}^{2} \mid \widetilde{\bar{S} \mid \overline{\boldsymbol{S}}}, \\
& \mathbf{L}^{\prime}=\widetilde{\bar{\theta} \overline{\mathbf{u}}}-\widetilde{\bar{\theta}} \widetilde{\overline{\mathbf{u}}}, \quad \mathbf{M}^{\prime}=\bar{\delta}^{2} \widetilde{\overline{\boldsymbol{S}} \nabla \bar{\theta}}-\widetilde{\bar{\delta}} \widetilde{\boldsymbol{S}} \widetilde{\nabla} \bar{\theta} .
\end{aligned}
$$

The tilde symbolizes a test filtering, with a width $\widetilde{\bar{\delta}} \geq \bar{\delta}$.

- Another model, which introduces the buoyancy term, is the Eidson model ([4]):

$$
\boldsymbol{T}^{d}=-2 C_{E} \bar{\delta}^{2} \sqrt{|\overline{\boldsymbol{S}}|^{2}-\frac{\beta g}{P r_{s g}} \frac{\partial \bar{\theta}}{\partial x_{3}}} \overline{\boldsymbol{S}}, \quad \mathbf{h}=-\frac{C_{E} \bar{\delta}^{2}}{P r_{s g}} \sqrt{|\overline{\boldsymbol{S}}|^{2}-\frac{\beta g}{P r_{s g}} \frac{\partial \bar{\theta}}{\partial x_{3}}} \nabla \bar{\theta},
$$

where $C_{E}$ is a constant. In practice, when the term under the radical is negative, the model is put to zero.

- To avoid the zero setting, Peng and Davidson ([21]) propose a modified version of the Eidson model:

$$
\begin{gathered}
\boldsymbol{T}^{d}=-2 C_{E} \bar{\delta}^{2} \frac{1}{|\overline{\boldsymbol{S}}|}\left(|\overline{\boldsymbol{S}}|^{2}-\frac{\beta g}{P r_{s g}} \frac{\partial \bar{\theta}}{\partial x_{3}}\right) \overline{\boldsymbol{S}} \\
\mathbf{h}=-\frac{C_{E} \bar{\delta}^{2}}{P r_{s g}} \frac{1}{|\overline{\boldsymbol{S}}|}\left(|\overline{\boldsymbol{S}}|^{2}-\frac{\beta g}{P r_{s g}} \frac{\partial \bar{\theta}}{\partial x_{3}}\right) \nabla \bar{\theta}
\end{gathered}
$$

- Another model, based on the scale-similarity hypothesis, is the scale-similarity model, adapted from Bardina model to the non-isothermal case:

$$
\boldsymbol{T}^{d}=\widetilde{\overline{\mathbf{u}} \otimes \overline{\mathbf{u}}}-\widetilde{\overline{\mathbf{u}}} \otimes \widetilde{\overline{\mathbf{u}}}, \quad \mathbf{h}=\widetilde{\bar{\theta} \overline{\mathbf{u}}}-\widetilde{\bar{\theta}} \widetilde{\mathbf{u}} .
$$


- The scale similarity hypothesis can be used to obtain others models which are combined with the Smagorinsky model to give a mixed model in the following form $([1,8,32,11])$ :

$$
\boldsymbol{T}^{d}=\boldsymbol{T}_{s}^{d}-2\left(C_{s} \bar{\delta}\right)^{2}|\overline{\boldsymbol{S}}| \overline{\boldsymbol{S}}, \quad \text { and } \quad \mathbf{h}=\mathbf{h}_{s}-\frac{\left(C_{s} \bar{\delta}\right)^{2}}{P r_{s g}}|\overline{\boldsymbol{S}}| \nabla \bar{\theta} .
$$

In the point of view of the symmetries, these models behave generally in the same way; so, we study only the following generic model:

$$
\boldsymbol{T}^{d}=(\widetilde{\overline{\mathbf{u}} \otimes \overline{\mathbf{u}}}-\widetilde{\overline{\mathbf{u}}} \otimes \widetilde{\mathbf{u}})^{d}-2\left(C_{s} \bar{\delta}\right)^{2}|\overline{\boldsymbol{S}}| \overline{\boldsymbol{S}}, \quad \text { and } \quad \mathbf{h}=\widetilde{\bar{\theta} \overline{\mathbf{u}}}-\widetilde{\bar{\theta}} \widetilde{\overline{\mathbf{u}}}-\frac{\left(C_{s} \bar{\delta}\right)^{2}}{P r_{s g}}|\overline{\boldsymbol{S}}| \nabla \bar{\theta} .
$$

Notice that the Eidson model seems not to have been used for many years. However, it is studied here because the analysis of this model could be used as a basis for the analysis of other models which are or are not presented in this paper.

The analysis of the models is done in the following way.

The set of the solutions $(\mathbf{u}, p, \theta)$ of $(1)$ is preserved by each of the symmetries cited above. We then require this to the set of the solutions $(\overline{\mathbf{u}}, \bar{p}, \bar{\theta})$ of $(28)$ too, since $(\overline{\mathbf{u}}, \bar{p}, \bar{\theta})$ is expected to be a good approximation of $(\mathbf{u}, p, \theta)$. More clearly, if a transformation

$T:(t, \mathbf{x}, \mathbf{u}, p, \theta) \mapsto(\widehat{t}, \widehat{\mathbf{x}}, \widehat{\mathbf{u}}, \widehat{p}, \widehat{\theta})$

is a symmetry of (1), we require that the model is such that the same transformation, applied to the filtered quantities,

$T:(t, \mathbf{x}, \overline{\mathbf{u}}, \bar{p}, \bar{\theta}) \mapsto(\widehat{t}, \widehat{\mathbf{x}}, \widehat{\mathbf{u}}, \widehat{\bar{p}}, \widehat{\bar{\theta}})$,

is a symmetry of the filtered equations (28). When this condition holds, the model is said to be invariant under the symmetry. If the model is invariant under all the symmetries, we can expect to retrieve the properties of (1), such as conservation laws, wall laws, exact solutions, spectrum properties, ... when approximating $(\mathbf{u}, p, \theta)$ by $(\overline{\mathbf{u}}, \bar{p}, \bar{\theta})$.

The presented models will be analyzed according to their invariance under each of the symmetries of (1). It will be assumed that the filter does not destroy the symmetry properties of the equations. Oberlack deduced in [16] an example of such a filter. Its kernel has the following form:

$G(\mathbf{x})=\frac{s+3}{4 \pi \ell^{s+3}}\|\mathbf{x}\|^{s} \mathbf{1}_{B(\ell)}(\mathbf{x})$

where $s$ is a real number greater than $3, \ell \in \mathbb{R}$ and $B(\ell)$ the centered ball of radius $\ell$.

In what follows, each category of symmetries will be taken one by one, and we investigate which models are invariant under the symmetries within the considered category.

\subsection{Time, pressure and Galilean translations}

All the above models are invariant under the time and the pressure translations (19) and (20) because neither $t$ nor $p$ is explicitly present in their expressions.

Applied to the filtered variables, the generalized Galilean transformations (23) is:

$(t, \mathbf{x}, \overline{\mathbf{u}}, \bar{p}, \bar{\theta}) \mapsto(\widehat{t}, \widehat{\mathbf{x}}, \widehat{\overline{\mathbf{u}}}, \widehat{\bar{p}}, \widehat{\bar{\theta}})=(t, \mathbf{x}+\boldsymbol{\alpha}(t), \overline{\mathbf{u}}+\dot{\boldsymbol{\alpha}}(t), \bar{p}+\rho \mathbf{x} \cdot \ddot{\boldsymbol{\alpha}}(t), \bar{\theta})$.

- Since $\widehat{\nabla \overline{\mathbf{u}}}=\nabla \overline{\mathbf{u}}$ and $\widehat{\bar{\theta}}=\bar{\theta}$, the Smagorinsky (29) and the Eidson (32)-(33) models are invariant under the generalized Galilean transformations. 
- For the scale-similarity model (34), we have:

$\widetilde{\widehat{\overline{\mathbf{u}}} \otimes \widehat{\widehat{\mathbf{u}}}}-\widetilde{\widehat{\mathbf{u}}} \otimes \widetilde{\widehat{\mathbf{u}}}=(\overline{\mathbf{u}}+\widetilde{\boldsymbol{\alpha}}) \otimes(\overline{\mathbf{u}}+\dot{\boldsymbol{\alpha}})-(\widetilde{\overline{\mathbf{u}}+\dot{\boldsymbol{\alpha}}}) \otimes(\widetilde{\overline{\mathbf{u}}+\dot{\boldsymbol{\alpha}}})=\widetilde{\overline{\mathbf{u}} \otimes \overline{\mathbf{u}}}-\widetilde{\mathbf{u}} \otimes \widetilde{\mathbf{u}}$

and

$\widetilde{\bar{\theta}} \widehat{\overline{\mathbf{u}}}-\widetilde{\bar{\theta}} \widetilde{\widehat{\mathbf{u}}}=\widetilde{\theta}(\overline{\mathbf{u}}+\dot{\boldsymbol{\alpha}})-\widetilde{\bar{\theta}}(\widetilde{\overline{\mathbf{u}}+\dot{\boldsymbol{\alpha}})}=\widetilde{\bar{\theta}} \overline{\mathbf{u}}-\widetilde{\bar{\theta}} \widetilde{\overline{\mathbf{u}}}$.

The model is then invariant under the generalized Galilean transformations.

- Next, since $\widehat{\overline{\boldsymbol{S}}}=\overline{\boldsymbol{S}}$ and $\widehat{\nabla \theta}=\nabla \theta$, we can write that

$\widehat{M}=M, \quad \widehat{\mathrm{M}}^{\prime}=\mathrm{M}^{\prime}$,

and from (41) and (42) that

$\widehat{\mathbf{L}}=\boldsymbol{L}, \quad \widehat{\mathbf{L}}^{\prime}=\mathbf{L}^{\prime}$.

The invariance of the dynamic model (30) follows from these expressions.

- Lastly, the mixed model (36) is generalized-Galilean invariant like the Smagorinsky and the scalesimilarity models.

All the models are then invariant under the three symmetry groups composed by the time, the pressure and the generalized Galilean transformations.

\subsection{Pressure-temperature translations}

The filtered equations (28) are invariant under the pressure-temperature translations

$(t, \mathbf{x}, \overline{\mathbf{u}}, \bar{p}, \bar{\theta}) \mapsto(\widehat{t}, \widehat{\mathbf{x}}, \widehat{\mathbf{u}}, \widehat{\bar{p}}, \widehat{\bar{\theta}})=\left(t, \mathbf{x}, \overline{\mathbf{u}}, \bar{p}+a \beta g x_{3}, \bar{\theta}+a \frac{1}{\rho}\right)$

if and only if $\widehat{\boldsymbol{T}}=\boldsymbol{T}$ and $\widehat{\mathbf{h}}=\mathbf{h}$.

- The Smagorinsky and the Eidson models (29), (32) and (33) are invariant under the pressuretemperature translations because $\widehat{\widehat{S}}=\overline{\boldsymbol{S}}$ and $\widehat{\bar{\theta}}=\bar{\theta}$.

- Next,

$$
\begin{aligned}
& \widetilde{\widehat{\mathbf{u}} \otimes \widehat{\overline{\mathbf{u}}}}-\widetilde{\widehat{\mathbf{u}}} \otimes \widetilde{\widehat{\mathbf{u}}}=\widetilde{\overline{\mathbf{u}} \otimes \overline{\mathbf{u}}}-\widetilde{\mathbf{u}} \otimes \widetilde{\mathbf{u}}, \quad \text { and } \\
& \widetilde{\widehat{\bar{\theta}}} \widehat{\overline{\mathbf{u}}}-\widetilde{\bar{\theta}} \widetilde{\overline{\mathbf{u}}}=\left(\widetilde{\bar{\theta}} \overline{\mathbf{u}}+a_{\rho}^{1} \widetilde{\widetilde{\mathbf{u}}}\right)-\left(\widetilde{\bar{\theta}} \widetilde{\mathbf{u}}+a \frac{1}{\rho} \widetilde{\mathbf{u}}\right)=\widetilde{\bar{\theta}} \overline{\mathbf{u}}-\widetilde{\bar{\theta}} \widetilde{\overline{\mathbf{u}}}
\end{aligned}
$$

The scale-similarity model (34) is then invariant.

- For the dynamic model (30), $\boldsymbol{L}, \boldsymbol{M}$ and $\mathbf{L}^{\prime}$ are unchanged. And since $\widehat{\nabla \theta}=\nabla \theta, \mathbf{M}^{\prime}$ remains also unchanged. The model is then invariant.

- At last, the invariance of the Smagorinsky and the scale-similarity models leads to the invariance of the mixed model (36) under the pressure-temperature translations. 


\subsection{Reflections and rotations}

Let us first consider the reflections about the horizontal coordinate axes $\mathbf{e}_{1}$ and $\mathbf{e}_{2}$, the horizontal (constant) rotations and the horizontal 2D material indifference. The reflection about the vertical axis $\mathbf{e}_{3}$ will be treated separately because it modifies the temperature variable $\theta$. The transformations, applied to the filtered variables, are then

$(t, \mathbf{x}, \overline{\mathbf{u}}, \bar{p}, \bar{\theta}) \mapsto(\widehat{t}, \widehat{\mathbf{x}}, \widehat{\overline{\mathbf{u}}}, \widehat{\bar{p}}, \widehat{\bar{\theta}})=\left(t, \boldsymbol{\Upsilon}_{\mathbf{x}}, \boldsymbol{\Upsilon}_{\overline{\mathbf{u}}}, \bar{p}, \bar{\theta}\right)$

where $\boldsymbol{\Upsilon}=\boldsymbol{R}$ or $\boldsymbol{\Lambda}$ with $\iota_{3}=1$.

Equations (28) are invariant if and only if

$\widehat{\boldsymbol{T}}=\boldsymbol{\Upsilon} \boldsymbol{T}{ }^{T} \boldsymbol{\Upsilon} \quad$ and $\quad \widehat{\mathbf{h}}=\boldsymbol{\Upsilon} \mathbf{h}$.

- It is straight forward to show that

$$
\widehat{\overline{\boldsymbol{S}}}=\boldsymbol{\Upsilon} \overline{\boldsymbol{S}}^{T} \boldsymbol{\Upsilon} \quad \text { and } \quad \widehat{\nabla} \widehat{\bar{\theta}}=\boldsymbol{\Upsilon \nabla} \bar{\theta} .
$$

The Smagorinsky and the Eidson models (29), (32) and (33) are then invariant under the two first reflections and the horizontal rotations.

- Moreover,

$$
\widehat{\overline{\mathbf{u}}} \otimes \widehat{\overline{\mathbf{u}}}=\boldsymbol{\Upsilon}(\overline{\mathbf{u}} \otimes \overline{\mathbf{u}})^{T} \boldsymbol{\Upsilon} \quad \text { and } \quad \widehat{\bar{\theta}} \overline{\overline{\mathbf{u}}}=\boldsymbol{r}(\bar{\theta} \overline{\mathbf{u}}) .
$$

This leads to the invariance of the scale-similarity model (34).

- For the dynamic model (30), we have

$$
\widehat{\boldsymbol{L}}=\boldsymbol{\Upsilon} \boldsymbol{L}^{T} \boldsymbol{\Upsilon}, \quad \widehat{\boldsymbol{M}}=\boldsymbol{\Upsilon} \boldsymbol{M}^{T} \boldsymbol{\Upsilon}, \quad \widehat{\mathbf{L}^{\prime}}=\boldsymbol{\Upsilon} \mathbf{L}^{\prime}, \quad \widehat{\mathbf{M}^{\prime}}=\boldsymbol{\Upsilon} \mathbf{M}^{\prime}
$$

Hence, $\widehat{C_{\boldsymbol{T}}}=C_{\boldsymbol{T}}$ and $\widehat{C_{\mathbf{h}}}=C_{\mathbf{h}}$. Consequently, this model verifies (46).

- Finally, relations (47) and (48) lead to the invariance of the mixed model (36).

All the models are invariant under the horizontal rotations and the two first reflections. Let us now consider the reflection in the third direction. The filtered equations (28) are invariant if and only if

$\widehat{\boldsymbol{T}}=\boldsymbol{\Lambda}_{3} \boldsymbol{T}^{T} \boldsymbol{\Lambda}_{3} \quad$ and $\quad \widehat{\mathbf{h}}=-\boldsymbol{\Lambda}_{3} \mathbf{h}$.

where $\boldsymbol{\Lambda}_{3}$ is the diagonal matrix $\boldsymbol{\Lambda}_{3}=\operatorname{diag}(1,1,-1)$.

- For this reflection, we have:

$\widehat{\overline{\boldsymbol{S}}}=\overline{\boldsymbol{S}} \quad$ and $\quad \widehat{\nabla} \widehat{\bar{\theta}}=-\boldsymbol{\Lambda}_{3} \nabla \bar{\theta}$.

These relations imply the invariance of the Smagorinsky model (29).

- In addition,

$$
\frac{\partial \widehat{\bar{\theta}}}{\partial \widehat{x}_{3}}=\frac{\partial \bar{\theta}}{\partial x_{3}} .
$$

Thus, the Eidson models verify (50) and (33) are also invariant under the third reflection. 
- For the scale-similarity model (34),

$$
\widehat{\overline{\mathbf{u}}} \otimes \widehat{\mathbf{u}}=\overline{\mathbf{u}} \otimes \overline{\mathbf{u}} \quad \text { and } \quad \widehat{\bar{\theta}} \widehat{\overline{\mathbf{u}}}=-\boldsymbol{\Lambda}_{3} \bar{\theta} \overline{\mathbf{u}} .
$$

This model is then invariant.

- Next, for the dynamic model (30),

$$
\widehat{\boldsymbol{L}}=L, \quad \widehat{M}=M, \quad \widehat{\mathbf{L}}^{\prime}=-\boldsymbol{\Lambda}_{3} \mathbf{L}^{\prime}, \quad \widehat{\mathbf{M}^{\prime}}=-\Lambda_{3} \mathbf{M}^{\prime}
$$

Hence, $\widehat{C_{\boldsymbol{T}}}=C_{\boldsymbol{T}}$ and $\widehat{C_{\mathbf{h}}}=C_{\mathbf{h}}$. It follows that the dynamic model is invariant under the third reflection.

- Lastly, the invariance of the mixed model (36) under the third reflection follows from the invariance of the Smagorinsky and the scale-similarity models.

All the models are invariant under the horizontal rotations and the reflections. The last symmetries of the non-isothermal Navier-Stokes equations are the scaling transformations.

\subsection{Scaling transformations}

The two scaling transformations can be combined into the following two-parameter scaling transformations:

$(t, \mathbf{x}, \mathbf{u}, p, \theta, \nu, \kappa) \mapsto\left(\mathrm{e}^{2 a} t, \mathrm{e}^{b+a} \mathbf{x}, \mathrm{e}^{b-a} \mathbf{u}, \mathrm{e}^{2 b-2 a} p, \mathrm{e}^{b-3 a} \theta, \mathrm{e}^{2 b} \nu, \mathrm{e}^{2 b} \kappa\right)$.

The first scaling transformations correspond to $b=0$ and the second ones to $a=0$. The filtered equations (28) are invariant if and only if

$\widehat{\boldsymbol{T}}=\mathrm{e}^{2 b-2 a} \boldsymbol{T} \quad$ and $\quad \widehat{\mathbf{h}}=\mathrm{e}^{2 b-4 a} \mathbf{h}$.

- Since $\widehat{\overline{\boldsymbol{S}}}=\mathrm{e}^{-2 a} \overline{\boldsymbol{S}}$ and $\widehat{\nabla \bar{\theta}}=\mathrm{e}^{-4 a} \nabla \bar{\theta}$, we have, for the Smagorinsky model (29):

$\widehat{\boldsymbol{T}}=\mathrm{e}^{-4 a} \boldsymbol{T} \quad$ and $\quad \widehat{\mathbf{h}}=\mathrm{e}^{-6 a} \mathbf{h}$.

The model verifies (56) neither when $a=0$ nor when $b=0$. Thus, it is invariant neither under the first nor under the second scaling transformations.

- The first terms of the Eidson models (32)-(33) are similar to the Smagorinsky model. These models are not invariant under the scaling transformations.

- The scale-similarity model (34) is invariant under the two types of scaling transformations because $\widehat{\widehat{\hat{\mathbf{u}}} \otimes \widehat{\widehat{\mathbf{u}}}}-\widetilde{\widehat{\mathbf{u}}} \otimes \widetilde{\widehat{\mathbf{u}}}=\mathrm{e}^{2 b-2 a}(\widetilde{\overline{\mathbf{u}} \otimes \overline{\mathbf{u}}}-\widetilde{\overline{\mathbf{u}}} \otimes \widetilde{\overline{\mathbf{u}}}) \quad$ and

$\widetilde{\bar{\theta}} \widehat{\overline{\mathbf{u}}}-\widetilde{\bar{\theta}} \widetilde{\widehat{\mathbf{u}}}=\mathrm{e}^{2 b-4 a}(\widetilde{\bar{\theta} \overline{\mathbf{u}}}-\widetilde{\bar{\theta}} \widetilde{\overline{\mathbf{u}}})$.

Condition (56) is verified.

- For the dynamic model (30), we have:

$\widehat{\boldsymbol{L}}=\mathrm{e}^{2 b-2 a} \boldsymbol{L}, \quad \widehat{\boldsymbol{M}}=\mathrm{e}^{-4 a} \boldsymbol{M}, \quad \widehat{\mathbf{L}^{\prime}}=\mathrm{e}^{2 b-4 a} \mathbf{L}^{\prime}, \quad \widehat{\mathbf{M}^{\prime}}=\mathrm{e}^{-6 a} \mathbf{M}^{\prime}$.

This implies that

$\widehat{C_{\boldsymbol{T}}}=\mathrm{e}^{2 b+2 a} C_{\boldsymbol{T}}, \quad \widehat{C_{\mathbf{h}}}=\mathrm{e}^{2 b+2 a} C_{\mathbf{h}}$.

Thus,

$\widehat{\boldsymbol{T}}=\mathrm{e}^{2 b-2 a} \boldsymbol{T} \quad$ and $\quad \widehat{\mathbf{h}}=\mathrm{e}^{2 b-4 a} \mathbf{h}$.

The dynamic model is invariant. 


\begin{tabular}{|l|c|c|c|c|}
\hline & $\begin{array}{c}\text { Time, pressure, } \\
\text { Galilean }\end{array}$ & Pressure-temperature & $\begin{array}{c}\text { Rotation, } \\
\text { reflection, } \\
\text { material }\end{array}$ & Scaling \\
\hline Smagorinsky & invariant & invariant & invariant & non-invariant \\
\hline Dynamic & invariant & invariant & invariant & invariant \\
\hline Eidson & invariant & invariant & invariant & non-invariant \\
\hline Modified Eidson & invariant & invariant & invariant & non-invariant \\
\hline Similarity & invariant & invariant & invariant & invariant \\
\hline Mixed & invariant & invariant & invariant & non-invariant \\
\hline
\end{tabular}

Table 1 Result of the model analysis

- At last, the mixed model (36) is not invariant under the scaling transformations because of the Smagorinsky part. However, the (Leonard) terms which correspond to the scale-similarity model are invariant.

To sum up, only the similarity and the dynamic models are invariant under the scaling transformations. The scaling transformations have a particular importance though because, for example, Oberlack used them to obtain wall laws which was validated in [18], [17] and [14]. Hence, a model which breaks the scaling symmetries cannot capture these wall laws. As mentioned by Oberlack in [16], the inability of the Smagorinsky model to properly represent the near-wall behaviour of the flow can be related to its non-invariance under the scaling transformations. In the same way, any solution which is self-similar according to one or both of the scaling transformations cannot be reproduced by a non-invariant model $([26])$. In addition, the conservation laws related to these symmetries are violated.

Table 1 summarizes the result of the analysis. It can be observed that many models are not invariant under the symmetry group of the equations. Only the dynamic and the scale-similarity models are invariant. The mixed model which is studied here is not invariant under the scaling transformation because of the Smagorinsky part. However, the mixing of a scale similarity type model with the dynamic model could, of course, lead to other invariant models.

\section{Conclusion}

The analysis done in this paper completes the work done in ([16]) and ([24]) on the Navier-Stokes equations. The calculation can easily be extended to the equation of a passive scalar.

As in the isothermal case, we showed that only the similarity and the dynamic models are invariant under the symmetry group of the non-isothermal Navier-Stokes equations. In fact, if we do not look at the test filter (which was supposed not to destroy the invariance properties), the scale-similarity model has the same mathematical structure as the actual subgrid stress tensor. Hence, it is not surprising that it is invariant. What is more interesting is the invariance of the dynamic model. In fact, the dynamic evaluation of constants restores the scale invariance of any model (as well for $\boldsymbol{T}$ as for $\mathbf{h}$ ).

However, these two invariant models are not always satisfactory. In particular, they use a test filtering which was supposed to be compatible with all the symmetries of the equations. This is not the case of all filters. In addition, the dynamic model is not conform to the second law of thermodynamics 
which not only is an important physical property but also a sufficient condition for the numerical stability $([23,26])$.

Other ways to develop invariant models are then needed. This can be done by extending the work done in $[25,24]$ on deriving invariant subgrid stress tensor models for isothermal fluid flows. In these articles, a class of subgrid stress tensor models which are invariant under the symmetry group of the Navier-Stokes equations and which are conform to the second law of thermodynamics were built. One of these models was then tested numerically to the configuration of a ventilated room. A better result than those provided by the Smagorinsky and the dynamic models was obtained.

\section{A Symmetries of the convection-diffusion equation}

In this section, we calculate "by hand" the symmetries of the convection-diffusion equation (62). The aim is to present briefly the procedure used to calculate the symmetries of an equation.

Consider the following one-dimensional convection-diffusion equation:

$\frac{\partial \theta}{\partial t}+u \frac{\partial \theta}{\partial x}-\kappa \frac{\partial^{2} \theta}{\partial x^{2}}=0$

where $u$ is a parameter. In order to find the one-parameter symmetries of (62), we must calculate the infinitesimal generators

$\mathbf{X}=X^{1} \frac{\partial}{\partial t}+X^{2} \frac{\partial}{\partial x}+X^{3} \frac{\partial}{\partial \theta}$

where the unknowns $X^{1}, X^{2}$ and $X^{3}$ depend on $t, x$ and $\theta$. To be used with equation (62), $\mathbf{X}$ must be prolonged into a vector field $\operatorname{pr}(\mathbf{X})$ which acts on the derivatives of $\theta$. According to Lie's theory $([19,9])$, this vector field has the following expression:

$\operatorname{pr}(\mathbf{X})=X^{1} \frac{\partial}{\partial t}+X^{2} \frac{\partial}{\partial x}+X^{3} \frac{\partial}{\partial \theta}+X^{t} \frac{\partial}{\partial \theta_{t}}+X^{x} \frac{\partial}{\partial \theta_{x}}+X^{x x} \frac{\partial}{\partial \theta_{x x}}$

where $\theta_{t}, \theta_{x}$ and $\theta_{x x}$ are the derivatives of $\theta$ and $X^{t}, X^{x}$ and $X^{x x}$ are defined by (to be compared with (6)):

$X^{t}=\left.\frac{\partial \widehat{\theta_{t}}}{\partial a}\right|_{a=0}, \quad X^{x}=\left.\frac{\partial \widehat{\theta_{x}}}{\partial a}\right|_{a=0}, \quad X^{x x}=\left.\frac{\partial \widehat{\theta_{x x}}}{\partial a}\right|_{a=0}$,

$a$ being the parameter of the transformation. Using a Taylor developpement, one obtains the following relations, if $D_{t}$ and $D_{x}$ represent respectively the total differentiation operators according to $t$ and $x$ :

$$
\begin{aligned}
& X^{t}=D_{t} X^{3}-\theta_{t} D_{t} X^{1}-\theta_{x} D_{t} X^{2}, \\
& X^{x}=D_{x} X^{3}-\theta_{t} D_{x} X^{1}-\theta_{x} D_{x} X^{2}, \\
& X^{x x}=D_{x} X^{x}-\theta_{x t} D_{x} X^{1}-\theta_{x x} D_{x} X^{2} .
\end{aligned}
$$

Once the prolonged vector field $\mathbf{p r}(\mathbf{X})$ introduced, the variables $t, x, \theta$ and each of the derivatives of $\theta$ are considered as independent variables (like new coordinates). $\mathbf{X}$ generates a symmetry of equation (62) if and only if

$\theta_{t}+u \theta_{x}-\kappa \theta_{x x}=0 \quad \Longrightarrow \quad \operatorname{pr}(\mathbf{X}) \cdot\left(\theta_{t}+u \theta_{x}-\kappa \theta_{x x}\right)=0$

The last equation of (67) gives:

$X^{t}+u X^{x}-\kappa X^{x x}=0$

Using (66), developping and replacing $\theta_{x x}$ by $\frac{1}{\kappa}\left(\theta_{t}+u \theta_{x}\right)$, condition (68) leads to the following system of PDE's: 


$$
\begin{aligned}
\frac{\partial X^{3}}{\partial t}+u \frac{\partial X^{3}}{\partial x}-\kappa \frac{\partial^{2} X^{3}}{\partial x^{2}} & =0 \\
-\frac{\partial X^{2}}{\partial t}+u \frac{\partial X^{2}}{\partial x}-2 \kappa \frac{\partial^{2} X^{3}}{\partial x \partial \theta}-\frac{\partial^{2} X^{2}}{\partial x^{2}} & =0 \\
\frac{\partial X^{3}}{\partial \theta}-\frac{\partial X^{1}}{\partial t}-u \frac{\partial X^{1}}{\partial x}+\kappa \frac{\partial^{2} X^{1}}{\partial x^{2}}-\frac{\partial X^{3}}{\partial \theta}+2 \frac{\partial X^{2}}{\partial x} & =0 \\
2 \frac{\partial X^{1}}{\partial \theta}+2 \kappa \frac{\partial^{2} X^{1}}{\partial x \partial \theta} & =0 \\
2 v \frac{\partial X^{2}}{\partial \theta}-\kappa \frac{\partial^{2} X^{3}}{\partial \theta^{2}}+2 \kappa \frac{\partial^{2} X^{2}}{\partial x \partial \theta} & =0 \\
\frac{\partial^{2} X^{2}}{\partial x^{2}} & =0 \\
\frac{\partial^{2} X^{1}}{\partial \theta^{2}} & =0 \\
\frac{\partial X^{1}}{\partial x} & =0 \\
\frac{\partial X^{1}}{\partial \theta} & =0 .
\end{aligned}
$$

After integration, equations (69)-(77) give the following forms of the components of $\mathbf{X}$ :

$$
\begin{array}{r}
X^{1}=a_{2} t^{2}+a_{1} t+a_{0}, \\
X^{2}=a_{2} t x+\frac{a_{1}}{2} x+\frac{b_{1}}{2} t+\frac{b_{0}}{2}, \\
X^{3}=\frac{1}{4 \kappa} \theta\left(-a_{2} x^{2}+2 u a_{2} t+u a_{1}-\frac{a_{2}}{2} t+c\right)+\theta_{0}(x, t),
\end{array}
$$

where the $a_{i}$ 's, $b_{i}$ 's and $c$ are arbitrary constants and $\theta_{0}$ an arbitrary solution of the convection-diffusion equation (62). $\mathbf{X}$ is then a linear combination of the following vector fields:

$$
\begin{array}{r}
\frac{\partial}{\partial t}, \\
\frac{\partial}{\partial x}, \\
\theta \frac{\partial}{\partial \theta}, \\
\theta_{0}(x, t) \frac{\partial}{\partial \theta}, \\
-2 \kappa t \frac{\partial}{\partial x}+(x-u t) \theta \frac{\partial}{\partial \theta}, \\
4 \kappa t \frac{\partial}{\partial t}+2 \kappa x \frac{\partial}{\partial x}+(x-u t) u \theta \frac{\partial}{\partial \theta}, \\
-4 \kappa t^{2} \frac{\partial}{\partial t}-4 \kappa x t \frac{\partial}{\partial x}+\left[(x-u t)^{2}+2 \kappa t\right] \theta \frac{\partial}{\partial \theta} .
\end{array}
$$

Each of these vector fields generates a symmetry of (62). The expressions of the symmetry transformations are deduced from the definition of an infinitesimal generator. For example, to obtain the symmetry transformation generated by the vector field (85), we solve the system

$\frac{\mathrm{d} \widehat{t}}{\mathrm{~d} a}=0, \quad \frac{\mathrm{d} \widehat{x}}{\mathrm{~d} a}=-2 \kappa \widehat{t}, \quad \frac{\mathrm{d} \widehat{\theta}}{\mathrm{d} a}=(\widehat{x}-u \widehat{t}) \widehat{\theta}$,

with the initial conditions

$\widehat{t}(a=0)=t, \quad \widehat{x}(a=0)=x, \quad \widehat{\theta}(a=0)=\theta$.

Equations (88) and (89) lead to

$\widehat{t}=t, \quad \widehat{x}=x-2 t \kappa a, \quad \widehat{\theta}=\theta \exp \left[(x-u t) a-t \kappa a^{2}\right]$. 
Using the same procedure for the other vector fields, we get the following symmetries of the convection-diffusion equation (62), corresponding respectively to the infinitesimal generators (81)-(87):

$$
\begin{aligned}
&(t, x, \theta) \longmapsto(t+a, x, \theta), \\
&(t, x, \theta) \longmapsto(t, x+a, \theta), \\
&(t, x, \theta) \longmapsto\left(t, x, \theta \mathrm{e}^{a}\right), \\
&(t, x, \theta) \longmapsto(t, x, \theta+\theta), \\
&(t, x, \theta) \longmapsto(t, x, \theta) \longmapsto\left(t, x-2 t \kappa a, \theta \exp \left[(x-u t) a-t \kappa a^{2}\right]\right), \\
&(t, x, \theta) \longmapsto\left(\frac{t}{1-4 t \kappa a}, \frac{x}{1-4 t \kappa a}, \theta \sqrt{1-4 t \kappa a} \exp \left[\frac{a(x-\kappa t)^{2}}{1-4 t \kappa a}\right]\right),
\end{aligned}
$$

where, as above, $\theta_{0}$ is any solution of $(62)$.

\section{References}

1. Bardina, J., Ferziger, J., Reynolds, W.: Improved turbulence models based on large eddy simulation of homogeneous, incompressible, turbulent flows. Tech. Rep. Report TF-19, Stanford University (1983)

2. Cannone, M.: Ondelettes, paraproduits et Navier-Stokes. Diderot Editeur, Arts et Sciences (1995)

3. Cantwell, B.: Similarity transformations for the two-dimensional, unsteady, stream-function equation. Journal of Fluid Mechanics 85, 257-271 (1978)

4. Eidson, T.: Numerical simulation of the turbulent Rayleigh-Bénard problem using subgrid modelling. Journal of Fluid Mechanics 158, 245-268 (1985)

5. Fureby, C., Tabor, G.: Mathematical and physical constraints on large-eddy simulations. Theorical and Computational Fluid Dynamics 9(2), 85-102 (1997)

6. Fushchych, W., Popowych, R.: Symmetry reduction and exact solutions of the Navier-Stokes equations I. Journal of Nonlinear Mathematical Physics 1(1), 75-113 (1994)

7. Hereman: Review of symbolic software for the computation of Lie symmetries of differential equations. Euromath Bulletin 1(2), 45-79 (1994)

8. Horiuti, K.: The role of the bardina model in large eddy simulation of turbulent channel flow. Physics of Fluids A 1(2), 426-428 (1989)

9. Ibragimov, N.: CRC handbook of Lie group analysis of differential equations. Vol 1: Symmetries, exact solutions and conservation laws. CRC Press (1994)

10. Ibragimov, N.: CRC handbook of Lie group analysis of differential equations. Vol 3: New trends in theorical developments and computational methods. CRC Press (1996)

11. Keating, A., Piomelli, U., Bremhorst, K., Nesic, S.: Large-eddy simulation of heat transfer downstream of a backward-facing step. Journal of Turbulence 5(020) (2004)

12. Kim, P., Olver, P.: Geometric integration via multi-space. Regular and Chaotic Dynamics 9(3), 213-226 (2004)

13. Lilly, D.: A proposed modification of the Germano subgrid-scale closure method. Physics of Fluids A4(3), 633-635 (1992)

14. Lindgren, B., Österlund, J., Johansson, A.: Evaluation of scaling laws derived from lie group symmetry methods in zero-pressure-gradient turbulent boundary layers. Journal of Fluid Mechanics 502, 127-152 (2004)

15. Nœther, E.: Invariante Variationsprobleme. In: Königliche Gesellschaft der Wissenschaften, pp. 235-257 (1918)

16. Oberlack, M.: Invariant modeling in large-eddy simulation of turbulence. In: Annual Research Briefs. Stanford University (1997)

17. Oberlack, M.: Symmetries, invariance and scaling-laws in inhomogeneous turbulent shear flows. Flow, Turbulence and Combustion 62(2), 111-135 (1999)

18. Oberlack, M., Cabot, W., Pettersson Reif, B., Weller, T.: Group analysis, direct numerical simulation and modelling of a turbulent channel flow with streamwise rotation. Journal of Fluid Mechanics 562, 355-381 (2006)

19. Olver, P.: Applications of Lie groups to differential equations. Graduate texts in mathematics. SpringerVerlag (1986)

20. Olver, P.: Geometric foundations of numerical algorithms and symmetry. Applicable Algebra in Engineering, Communication and Computing 11(5), 417-436 (2001) 
21. Peng, S.H., Davidson, L.: Comparison of subgrid-scale models in LES for turbulent convection flow with heat transfer. In: 2nd EF Conference in Turbulent Heat Transfer, vol. 1, pp. 5.25-5.35 (1998)

22. Pukhnachev, V.: Invariant solution of Navier-Stokes equations describing motions with free boundary. Doklady Akademii Nauk SSSR pp. 202-302 (1972)

23. Razafindralandy, D., Hamdouni, A.: Consequences of symmetries on the analysis and construction of turbulence models. Symmetry, Integrability and Geometry: Methods and Applications 2, Paper 052 (2006)

24. Razafindralandy, D., Hamdouni, A.: Symmetry invariant subgrid models. In: G.M. Lamballais Friedrich (ed.) Direct and Large-Eddy Simulation 6: Proceedings of the Sixth International ERCOFTAC Workshop on Direct and Large-Eddy Simulation. Springer (2006)

25. Razafindralandy, D., Hamdouni, A., Béghein, C.: A class of subgrid-scale models preserving the symmetry group of Navier-Stokes equations. Communications in Nonlinear Science and Numerical Simulation 12(3), 243-253 (2007)

26. Razafindralandy, D., Hamdouni, A., Oberlack, M.: Analysis and development of subgrid turbulence models preserving the symmetry properties of the Navier-Stokes equations. To appear in European Journal of Mechanics/B

27. Speziale, C.: Some interesting properties of two-dimensional turbulence. Physics of Fluids 24, 1425-1427 (1981)

28. Ünal, G.: Application of equivalence transformations to inertial subrange of turbulence. Lie Group and Their Applications 1(1), 232-240 (1994)

29. Vu, K., Carminati, J.: Symbolic computation and differential equations: Lie symmetries. Journal of Symbolic Computation 29(2), 95-116 (2000)

30. Wang, L.: Frame-indifferent and positive-denite Reynolds stress-strain relation. Journal of Fluid Mechanics 352, 341-358 (1997)

31. Wang, L.: Physics-preserving turbulent closure models: SGS flux vectors of mass and energy. In: $3^{\text {rd }}$ AFOSR International Conference on Direct Numerical Simulation and Large Eddy Simulation (TAICDL) (2001)

32. Winckelmans, G., Wray, A., Vasilyev, O.: Testing of a new mixed model for LES : the Leonard model supplemented by a dynamic Smagorinsky term. In: Summer Program, pp. 367-388. Center for Turbulence Research, NASA Ames/Standford University (1998) 